\title{
Productividad de Clones de cacao tipo Nacional en una zona del bosque húmedo Tropical de la PRovincia de Los Ríos, Ecuador
}

\section{Type Nacional cocoa clone productivity in an area of Tropical rainforest in Los Ríos, Ecuador}

${ }^{\bullet}$ Fernando Sánchez-Mora ${ }^{1,2}$, José Zambrano Montufar ${ }^{1}$, Jaime Vera Chang ${ }^{1}$, Rommel Ramos Remache ${ }^{1}$, Felipe Gárces Fiallos ${ }^{1}$, Gregório Vásconez Montúfar ${ }^{1}$

${ }^{1}$ Dirección de Investigación Cientifica y Tecnológica, Universidad Técnica Estatal de Quevedo. Campus Finca Experimental

"La Maria”, km 7 vía Quevedo-El Empalme.EC.120501. Mocache, Ecuador. nandoslipnot25@hotmail.es; jverac@uteq.edu.ec; rramos@uteq.edu.ec; felipegarces23@yahoo.com; goyitouteq@hotmail.com

${ }^{2}$ Facultad de Ingeniería Agronómica, Universidad Técnica de Manabi. Campos Experimental La Teodomira, km 13 1⁄2 via Santa Ana. Santa Ana, Ecuador. ${ }^{\circledR}$ fernandosanchezm23@hotmail.com

\section{RESUMEN}

$\mathrm{E}^{1}$ Ecuador se caracteriza por producir cacao fino de aroma, producto altamente apetecido por los mercados internacionales. Sin embargo, la baja producción de este tipo de cacao ha llevado a los agricultores a optar por otras variedades de mayor rentabilidad. Bajo esta perspectiva se evaluó el comportamiento agronómico de 150 clones experimentales de cacao tipo Nacional establecidos en la Finca Experimental "La Represa", de la Universidad Técnica Estatal de Quevedo, a fin de identificar clones con características superiores, como un aporte al mejoramiento productivo del cacao en el país. Para cada clon se estableció una parcela con 10 plantas, distribuidas en un marco de plantación de $3 \times 3 \mathrm{~m}$. Se evaluó el índice de mazorca, índice de almendra, mazorcas sanas (\%) y rendimiento de cacao ( $\left.\mathrm{kg} \mathrm{ha}^{-1} \mathrm{año}^{-1}\right)$. Los datos de 100 clones, que proporcionaron información completa, se analizaron a través de estadística descriptiva y multivariada. Los clones L32H72 y L46H70 registraron los mejores índices de mazorca con 14.6 y 15.3 mazorcas, respectivamente. Los clones L32H72, L33H27 y L45H11, se destacaron por obtener un índice de almendra entre 1.92 y $1.97 \mathrm{~g}$ almendra $^{-1}$ seca. Los clones L12H27, L17H30 y L20H48 presentaron porcentajes de mazorcas sanas $<90 \%$ y registraron los mejores rendimientos con producciones de 1820, $1154 \mathrm{y}$ $1092 \mathrm{~kg} \mathrm{ha}^{-1}$ año $^{-1}$, respectivamente. Los clones L12H27 y L17H30 obtuvieron una alta productividad, adecuada estabilidad de la producción y cierta tolerancia a las enfermedades del cacao. Estos clones experimentales tipo Nacional se presentan como promisorios para nuevos estudios agronómicos y pueden ser utilizados en programas de conservación y mejoramiento genético.

Palabras clave: Theobroma cacao, clones experimentales, producción de cacao, recursos genéticos.

Recibido: 18-mayo-2012. Recibido en forma corregida: 9-abril-2013.

Aceptado: 13-enero-2014.

Publicado como ARTÍCULO CIENTÍFICO en Ciencia y Tecnología 7(1): 33-41

Enero-Junio de 2014

ISSN 1390-4051 impreso; ISSN 1390-4043 electrónico

(C) Ciencia y Tecnología. UTEQ. Quevedo-Ecuador

\section{Abstract}

$\mathrm{E}$ cuador is known for producing fine aroma cocoa which is highly sought after in international markets. However, the low production of this type of cocoa has led farmers to opt for higher profitability varieties. From this perspective, an evaluation of the agronomic performance of 150 type Nacional cocoa clones established at La Represa experimental farm was made by Quevedo Technical State University (Ecuador), to identify healthier and more productive individuals and reintroduce them to farmers as a contribution to improving cocoa production in the country. For each clone, a 10 plant spread over a $3 \times 3$ m planting framework plot was established. The study considered four variables, namely the percentage of healthy pods, pods index, seed index and cocoa yield $\left(\mathrm{kg} \mathrm{ha}^{-1} \mathrm{yr}^{-1}\right)$. Data from 100 clones, which provided complete information is analyzed using descriptive and multivariate statistics. From cluster analysis two groups were determined where Group lincluded 29 experimental clones with greater similarity representing $19.3 \%$ of evaluated materials. Clones L32H72 and L46H70 showed excellent attributes regarding pods index with 14.6 and 15.3 pods $\mathrm{kg}^{-1}$ of dry cocoa respectively. Clones L32H72, L33H27 and L45-H11, showed the better seed index between 1.92 and 1.97 g seed $^{-1}$ dry. Clones L12H27, L17H30 and L20H48, showed percentage of healthy pods $<90 \%$ and dry cocoa yields were obtained with production of 1820, 1154 and $1092 \mathrm{~kg} \mathrm{ha}^{-1} \mathrm{yr}^{-1}$. L12H27 and L17H30 clones showed high productivity, adequate production stability of production and some tolerance to cocoa diseases. These experimental type Nacional clones are presented as promising for further agronomic studies and possible use in conservation and breeding and possible use in conservation and breeding.

Key words: Theobroma cacao, experimental clones, cocoa production, genetic resources 


\section{INTRODUCCIÓN}

$\mathrm{E}$ 1 Ecuador es el primer productor mundial de cacao (Theobroma cacao L.) de alta calidad, conocido en los mercados internacionales como Sabor Arriba, siendo uno de los principales cultivos de interés comercial en la región. Sin embargo, aún existen graves problemas en la producción de cacao debido al inadecuado y escaso manejo agronómico de las cacaoteras, como también el uso de materiales genéticos de baja productividad y alta susceptibilidad a enfermedades (Quiroz y Amores, 2002). Esto ha ocasionado que los agricultores sustituyan la variedad tradicional por materiales de origen Trinitario más productivos y tolerantes a enfermedades, ocasionando una erosión genética, especialmente de genes relacionados con la calidad.

En el país se estima un rendimiento promedio de $300 \mathrm{~kg} \mathrm{ha}^{-1} \mathrm{año}^{-1}$ a nivel de productor (Quiroz y Amores, 2002), manteniéndose por debajo del promedio mundial de $480 \mathrm{~kg} \mathrm{ha}^{-1}$ año $^{-1}$. Los bajos rendimientos del cacao Nacional se atribuyen al uso de genotipos de origen genético desconocido, sin seleccionar, mezclados y de poca productividad, además de la alta incidencia de patógenos del fruto como: moniliasis (Moniliophthora roreri (Cif y Par) Evans et al.), escoba de bruja (Moniliophthora perniciosa (Stahel) Aime \& PhillipsMora) y pudrición del fruto (Phytophthora spp.).

La moniliasis causa la pudrición de las mazorcas, siendo una de las enfermedades más destructivas de América Latina, de donde es endémica (Phillips-Mora et al., 2005; Phillips-Mora et al., 2007; Grisales y Afanador, 2007). Esta enfermedad causa pérdidas superiores al 50\% de la cosecha en plantaciones con bajo nivel de tecnología (Sánchez et al., 2003; SánchezMora y Garcés-Fiallos, 2012). Por su lado, la escoba de bruja es un patógeno que ha provocado grandes pérdidas económicas en todas las regiones de cultivo, donde ha causado reducciones en el rendimiento que van desde 50 a 90\% (Meinhardt et al., 2008; Almeida et al., 2009). La propagación de esta enfermedad amenaza a todos los países de América donde se cultiva cacao, constituyendo además una seria amenaza para los países libres de esta enfermedad (Pereira et al., 1996). La moniliasis y la escoba de bruja causan pérdidas mayores que las producidas por Phytophthora (Dantas-Neto et al., 2005).

Los esfuerzos para controlar las enfermedades, deben estar orientados en mejorar la calidad del material de siembra (Evans, 2007), sin descuidar la calidad de almendras del cacao, el propósito del mejoramiento de este cultivo es el aumento del rendimiento o producción de cacao seco (Carvalho et al., 2001). Sin embargo, difícilmente se puede encontrar características ideales de productividad y sanidad en un mismo árbol, por lo que es necesario evaluar colecciones de cacao para seleccionar genotipos de interés y por medio de una acción dirigida generar nuevos materiales genéticos. Es decir, que la selección de los genotipos más distantes entre sí, pueden constituir fuentes de diferentes alelos para la combinación y formación de cultivares agronómicamente superiores, a fin de reunir genes de interés en una misma planta (Leal et al., 2008; SánchezMora et al., 2012).

En base a los antecedentes descritos, el objetivo de este estudio fue identificar clones de alta productividad y con cierto grado de resistencia a las enfermedades moniliasis y escoba de bruja.

\section{Materiales y métodos}

Q1 estudio se realizó en la Finca Experimental LLa Represa, localizada en el km 7.5 de la vía Quevedo-San Carlos, provincia de Los Ríos, cuyas coordenadas geográficas son 79³0'23" longitud Oeste y $01^{\circ} 00^{\prime} 35^{\prime \prime}$ de latitud Sur, a una altura de $90 \mathrm{msnm}$. Las condiciones climáticas durante la investigación fueron las siguientes: Temperatura promedio de $25.2^{\circ}$ $\mathrm{C}$, precipitación media anual de $3032.1 \mathrm{~mm}$, humedad relativa de $84 \%$ y heliofanía de 758.2 horas/luz año-1, (anuario meteorológico de 2010 en el Instituto Nacional de Meteorología e Hidrología, INAMHI, Quito, Ecuador). La topografía del terreno es plana, de textura del suelo franco arcilloso y $\mathrm{pH}$ de 5.7, condiciones edafoclimáticas ideales para el cultivo de cacao (Enríquez, 2004).

Durante el año 2010, se evaluaron 150 clones experimentales de cacao tipo Nacional, de siete años de edad. Todos los materiales son originarios de la colección CCAT (Centro de cacao fino y de aroma Tenguel). Las parcelas en estudio estuvieron conformadas de 10 plantas por clon experimental, distribuidas en dos hileras, a una distancia de 3 × 3 m entre plantas, con una densidad poblacional de 1111 plantas por hectárea.

\section{Variables evaluadas}

Mazorcas sanas. El fruto de cacao en estado de maduración fisiológica y sin ninguna afectación visible de enfermedades, fue removido y contado mensualmente. Posteriormente se calculó el porcentaje de mazorcas sanas por cada clon experimental, mediante la fórmula: MS (\%)= NMS/NMT (100).

Índice de mazorcas (IM). Se refiere al número de mazorcas necesarias para obtener un $\mathrm{kg}$ de cacao seco al 7\% de humedad. Se determinó aplicando la fórmula: $\mathrm{IM}=\left(\mathrm{N}^{\mathrm{o}}\right.$ mazorcas $\left.\mathrm{x} 1000\right) /$ (Peso en gramos de las almendras secas de $\mathrm{N}^{\mathrm{o}}$ mazorcas). Para obtener este parámetro, se tomó al azar una muestra de diez 
mazorcas maduras y sanas por parcela, cuyas almendras se sometieron al respectivo proceso de fermentación y secado.

Índice de almendras (IS). Es el peso promedio, en gramos, de una almendra de cacao seco, al 7\% de humedad. De las mazorcas recolectadas para determinar el IM, se tomaron al azar 100 almendras y se calculó el IS, utilizando la fórmula: IS= Peso en gramos de 100 almendras fermentadas y secas / 100 .

Rendimiento de cacao seco por año $\left(\mathrm{kg} \mathrm{ha}^{-1}\right)$. Se calculó multiplicando el peso fresco anual del cacao por planta por 0.40 (factor de conversión para obtener el peso seco). Para obtener el valor promedio de peso fresco, se pesó, mensualmente, las almendras frescas extraídas de mazorcas sanas, utilizando una balanza de precisión.

\section{Manejo del cultivo durante el estudio}

Para el mantenimiento de la colección en estudio se realizó un control manual de malezas, de dos deshierbas (coronas) previo a la fertilización (inicios y final de la época lluviosa), y tres aplicaciones químicas de paraquat (Gramoxone ${ }^{\circledR}$ NF: $276 \mathrm{~g}$ de i.a.) en dosis de $1 \mathrm{~L} \mathrm{ha}^{-1}$. Se fertilizó con un abono completo compuesto de N, P, K, $\mathrm{Mg}$, S y B (18-6-22-3-4-0.53), a la entrada y salida de la época lluviosa (enero y mayo), en dosis de $400 \mathrm{~g}$ por planta. Se efectuaron también podas sanitarias al final de la época seca. Además, mensualmente se eliminaron brotes ortotrópicos (brotes basales provenientes del patrón de injerto). Una vez realizada la poda se procedió a desinfectar las heridas con pasta bordelés, la misma que estuvo compuesta por cal y óxi-cloruro de cobre (3:1). No se aplicó fungicida para el control de enfermedades, ni se realizaron riegos durante la época seca.

\section{Análisis estadístico}

Con los datos registrados en campo se seleccionaron 100 clones que proporcionaron valores completos, correspondientes al $66.7 \%$ de los 150 clones experimentales estudiados. Se elaboró una matriz mixta de las variables estudiadas. Para el análisis de los datos se utilizó la estadística descriptiva y multivariada. En el análisis de distribución de frecuencias, primeramente se calculó el rango $(\mathrm{R})$ y el número de intervalos de clase $\left(\mathrm{K}=\bigvee_{\mathrm{n}}\right)$. Posteriormente se calculó el tamaño de un intervalo de clase mediante la fórmula $\mathrm{C}=\mathrm{R} / \mathrm{K}$.

A partir de los 10 árboles de cada clon se calculó el promedio de rendimiento por hectárea y el porcentaje de mazorcas sanas de cada clon. A partir de esos árboles también se obtuvo la variabilidad intra clon, utilizando la fórmula:

$$
\text { Variabilidad }(\%)=\frac{\text { Error estándar }}{\text { Média aritmética }} \times 100
$$

Los análisis estadísticos fueron realizados en el programa estadístico PAST versión 2.17c (Hammer et al., 2001).

\section{Resultados Y Discusión}

G $\mathrm{n}$ el primer trimestre del año evaluado, la aparición de frutos enfermos fue menor en comparación al segundo y tercer trimestre, indicando que hubo un incremento de infecciones por patógenos en la fructificación del cacao en la época lluviosa, provocando un aumento de frutos enfermos en la época seca (Figura 1). Similarmente, González y Vega (1992) en Costa Rica, evaluaron la aparición de frutos enfermos por mes y encontraron que hubo pocos frutos enfermos en la primera mitad del año, pero en la segunda mitad se intensificaron las enfermedades que provocaron más frutos enfermos en septiembre que sanos en noviembre. También se observó que entre los meses de octubre a marzo se registró la mayor producción de cacao, en la zona de Quevedo, concordando con Maddison et al. (1995), quienes en esta misma zona encontraron que gran parte de la cosecha de frutos sanos se obtiene entre octubre y marzo de cada año, durante el período de cosecha de la época seca.

El análisis descriptivo de las variables permitió una visualización de la variabilidad existente en la colección de cacao. Se observó que los coeficientes de variación fluctuaron entre $16.31 \%$ para la variable índice de almendra y $70.09 \%$ para la variable rendimiento de cacao seco (Cuadro 1). Este comportamiento probablemente fue provocado por las características genotípicas de cada clon y su interacción con el medio ambiente. Los caracteres agronómicos con amplio coeficiente de variación son importantes para determinar niveles de variabilidad genética dentro de la especie $T$. cacao, ya que mientras mayor sean los niveles de variación mayor será el margen de acción natural o artificial (Peña, 2003).

Considerando el criterio de las mejores características productivas en las variables estudiadas, mediante el análisis de frecuencias se observó que, en el porcentaje de mazorcas sanas, el $16 \%$ de los clones estudiados registraron una sanidad $\geq 90 \%$. El $41 \%$ de los clones presentaron índices de mazorca entre $14.6 \mathrm{y}$ 21.2. En el índice de almendra, el $35 \%$ de los clones registraron índices del 1.47 al 1.97 gramos. El 2\% de los clones superaron los $1100 \mathrm{~kg}$ de cacao seco ha ${ }^{-1}$ (Cuadro 2). En todas las variables analizadas se presentaron clones con valores aceptables en cuanto a sanidad y producción, aunque el coeficiente de variación (CV\%) fue alto, lo que es un indicativo de alta variabilidad. En la variable índice de mazorca los clones experimentales: L32H72, L46H70, L19H32, L18H58, L46H75, L29H48, 


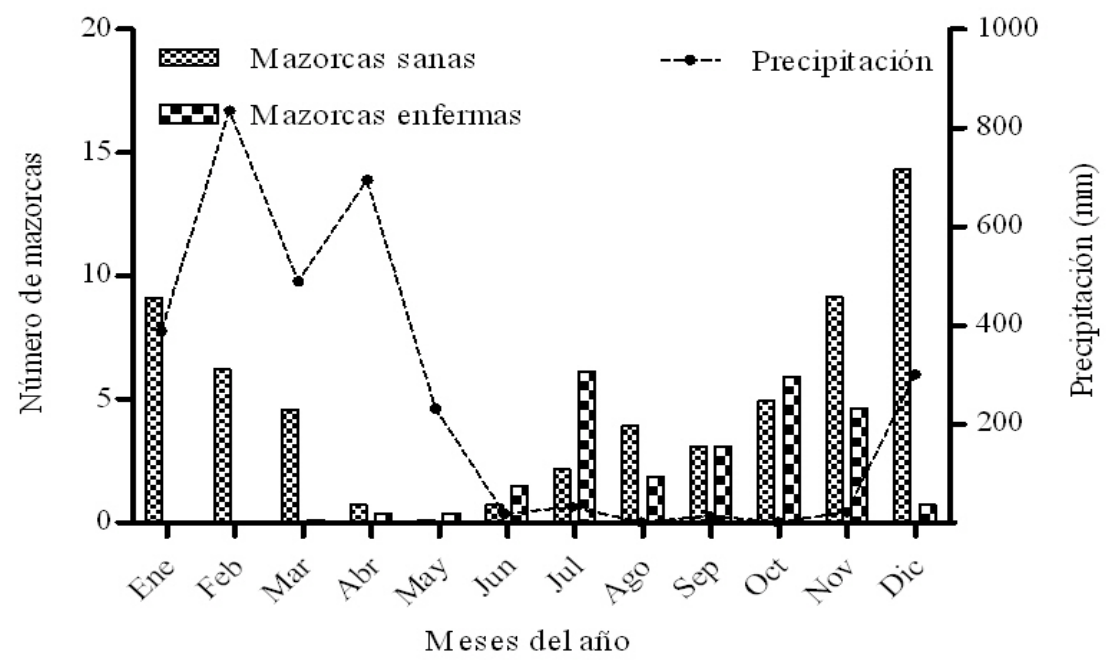

Figura 1. Promedio mensual del número de mazorcas sanas y enfermas cosechadas en la colección de $\mathbf{1 0 0}$ clones experimentales de cacao

Cuadro 1. Parámetros descriptivos de las variables mazorcas sanas (\%), índice de mazorca, índice de almendra y rendimiento de cacao seco registrados en 100 clones experimentales de cacao

\begin{tabular}{lcccc}
\hline \multicolumn{1}{c}{ Clones } & $\begin{array}{c}\text { Mazorcas sanas } \\
(\%)\end{array}$ & Índice de Mazorca & Índice de Almendra & $\begin{array}{c}\text { Rendimiento de } \\
\text { cacao seco } \\
\left(\mathbf{k g ~ h a}^{-1} \mathbf{~}\right.\end{array}$ \\
\hline Media & 77.31 & 23.30 & 1.39 & 376.02 \\
Mediana & 78.81 & 22.55 & 1.36 & 306.92 \\
Moda & 79.70 & 21.90 & 1.31 & - \\
Desviación estandar & 12.61 & 5.68 & 0.22 & 263.53 \\
Coeficiente de variación (\%) & 16.31 & 24.36 & 16.19 & 70.09 \\
Valores Mín. & 45.63 & 14.60 & 0.73 & 34.44 \\
Valores Máx. & 100.00 & 47.60 & 1.97 & 1820.05 \\
\hline
\end{tabular}

L46H88, L34H67, L18H59, L25H60, L30H25, L9H26, L33H27, L20H5, L8H12, L30H7 y L21H3, registraron índices entre 14.6 y 17.7 mazorcas (Cuadro 2).

Este índice es importante en la selección de clones productivos debido a que el número de mazorcas sanas por planta no siempre es un indicador de mejoramiento genético en términos de mayor rendimiento. Esto concuerda con Carvalho et al. (2001) quienes mencionan que a pesar de la relevancia de la producción de frutos, el peso de almendras es el carácter que más interesa al productor.

En la variable índice de almendra se destacaron los clones: L45H11, L33H27, L32H72, L46H68, L17H33, L18H58, L48H23, L20H48, L19H32, L46H71, L18H45, L20H5, L13H11, L46H67 y L41H70 con índices de 1.97 a $1.62 \mathrm{~g}$ almendra $^{-1}$ (Cuadro 2). Los altos índices de almendra son indicadores claves para el rendimiento anual y estudios de mejoramiento. Según Arciniegas (2005) los genotipos de cacao que tienen índices de almendra superiores a $1.0 \mathrm{~g}$ almendra ${ }^{-1}$ son aceptables desde el punto vista fitogenético e industrial.

Por medio del análisis de Componentes Principales, se explicó el total de la varianza de los datos obtenidos, a partir de las variables analizadas. Los dos primeros componentes principales explicaron el $73.02 \%$ de la variabilidad total. El primer componente explicó el $46.93 \%$ de la variabilidad, a través de las variables: porcentaje de mazorcas sanas (0.40) e índice de mazorca (0.64); mientras que el segundo componente explicó el $26.09 \%$ de la variabilidad, donde la variable de mayor relevancia fue el rendimiento de cacao (0.89) (Cuadro 3).

Estos resultados indican que estas variables permiten discriminar los clones de cacao en distintos 
Cuadro 2. Distribución de frecuencias de las variables mazorcas sanas (\%), índice de mazorca, índice de almendra y rendimiento de cacao seco registrado en 100 clones experimentales de cacao

\begin{tabular}{|c|c|c|c|c|c|c|c|}
\hline \multicolumn{4}{|c|}{ Mazorcas sanas (\%) } & \multicolumn{4}{|c|}{ Índice de mazorca } \\
\hline $\mathrm{N}^{0}$ Clase & Intervalo & $\begin{array}{l}\mathbf{N}^{\circ} \text { de } \\
\text { clones }\end{array}$ & $\begin{array}{l}\text { Porcentaje } \\
\text { acumulado }\end{array}$ & $\mathbf{N}^{0}$ Clase & Intervalo & $\begin{array}{l}N^{0} \text { de } \\
\text { clones }\end{array}$ & $\begin{array}{l}\text { Porcentaje } \\
\text { acumulado }\end{array}$ \\
\hline 1 & $45.63-51.07 \mid$ & 3 & 3 & 1 & $14.6-17.9$ & 17 & 17 \\
\hline 2 & $51.07-56.51 \mid$ & 4 & 7 & 2 & $17.9-21.21$ & 24 & 41 \\
\hline 3 & $56.51-61.95 \mid$ & 4 & 11 & 3 & $21.2-24.5$ & 27 & 68 \\
\hline 4 & $61.95-67.39$ & 12 & 23 & 4 & $24.5-27.8$ & 15 & 83 \\
\hline 5 & $67.39-72.83 \mid$ & 13 & 36 & 5 & $27.8-31.11$ & 7 & 90 \\
\hline 6 & $72.83-78.27 \mid$ & 12 & 48 & 6 & $31.1-34.4 \mid$ & 5 & 95 \\
\hline 7 & $78.27-83.71 \mid$ & 15 & 63 & 7 & $34.4-37.7 \mid$ & 4 & 99 \\
\hline 8 & $83.71-89.15$ & 21 & 84 & 8 & $37.7-41.0$ & 0 & 99 \\
\hline 9 & $89.15-94.59 \mid$ & 10 & 94 & 9 & $41.0-44.3 \mid$ & 0 & 99 \\
\hline \multirow[t]{2}{*}{10} & $94.59-100$ & 6 & 100 & 10 & $44.3-47.61$ & 1 & 100 \\
\hline & \multicolumn{3}{|c|}{100} & \multicolumn{4}{|c|}{100} \\
\hline \multicolumn{4}{|c|}{ Índice de almendra } & \multicolumn{4}{|c|}{ Rendimiento de cacao seco $\left(\mathrm{kg} \mathrm{ha}^{-1}\right)$} \\
\hline $\mathbf{N}^{0}$ Clase & Intervalo & $\begin{array}{l}\mathbf{N}^{0} \text { de } \\
\text { clones }\end{array}$ & $\begin{array}{l}\text { Porcentaje } \\
\text { acumulado }\end{array}$ & $\mathbf{N}^{0}$ Clase & Intervalo & $\begin{array}{l}N^{0} \text { de } \\
\text { clones }\end{array}$ & $\begin{array}{l}\text { Porcentaje } \\
\text { acumulado }\end{array}$ \\
\hline 1 & $0.73-0.85$ & 1 & 1 & 1 & $34.44-213.0$ & 29 & 29 \\
\hline 2 & $0.85-0.98$ & 3 & 4 & 2 & $213.0-391.56$ & 36 & 65 \\
\hline 3 & $0.98-1.10$ & 4 & 8 & 3 & $391.56-570.12$ & 17 & 82 \\
\hline 4 & $1.10-1.23$ & 17 & 25 & 4 & $570.12-748.681$ & 11 & 93 \\
\hline 5 & $1.23-1.35$ & 24 & 49 & 5 & $748.68-927.24 \mid$ & 3 & 96 \\
\hline 6 & $1.35-1.47 \mid$ & 16 & 65 & 6 & $927.24-1105.81$ & 2 & 98 \\
\hline 7 & $1.47-1.60$ & 20 & 85 & 7 & $1105.8-1284.36$ & 1 & 99 \\
\hline 8 & $1.60-1.72$ & 8 & 93 & 8 & $1284.36-1462.92 \mid$ & 0 & 99 \\
\hline 9 & $1.72-1.85 \mid$ & 4 & 97 & 9 & $1462.92-1641.48$ & 0 & 99 \\
\hline 10 & $1.85-1.97 \mid$ & 3 & 100 & 10 & $1641.48-1820.05$ & 1 & 100 \\
\hline
\end{tabular}

Cuadro 3. Componentes principales para las variables evaluadas en 100 clones experimentales de cacao

\begin{tabular}{cccc}
\hline $\begin{array}{c}\text { Componente } \\
\text { Principal }\end{array}$ & Autovalor & $\begin{array}{c}\text { Varianza total } \\
\text { (\%) }\end{array}$ & Variancia acumulada \\
\hline 1 & 1.88 & 46.93 & 46.93 \\
2 & 1.04 & 26.09 & 73.02 \\
3 & 0.78 & 19.50 & 92.52 \\
4 & 0.30 & 7.48 & 100.00 \\
\hline
\end{tabular}


Sánchez-Mora et al., 2014

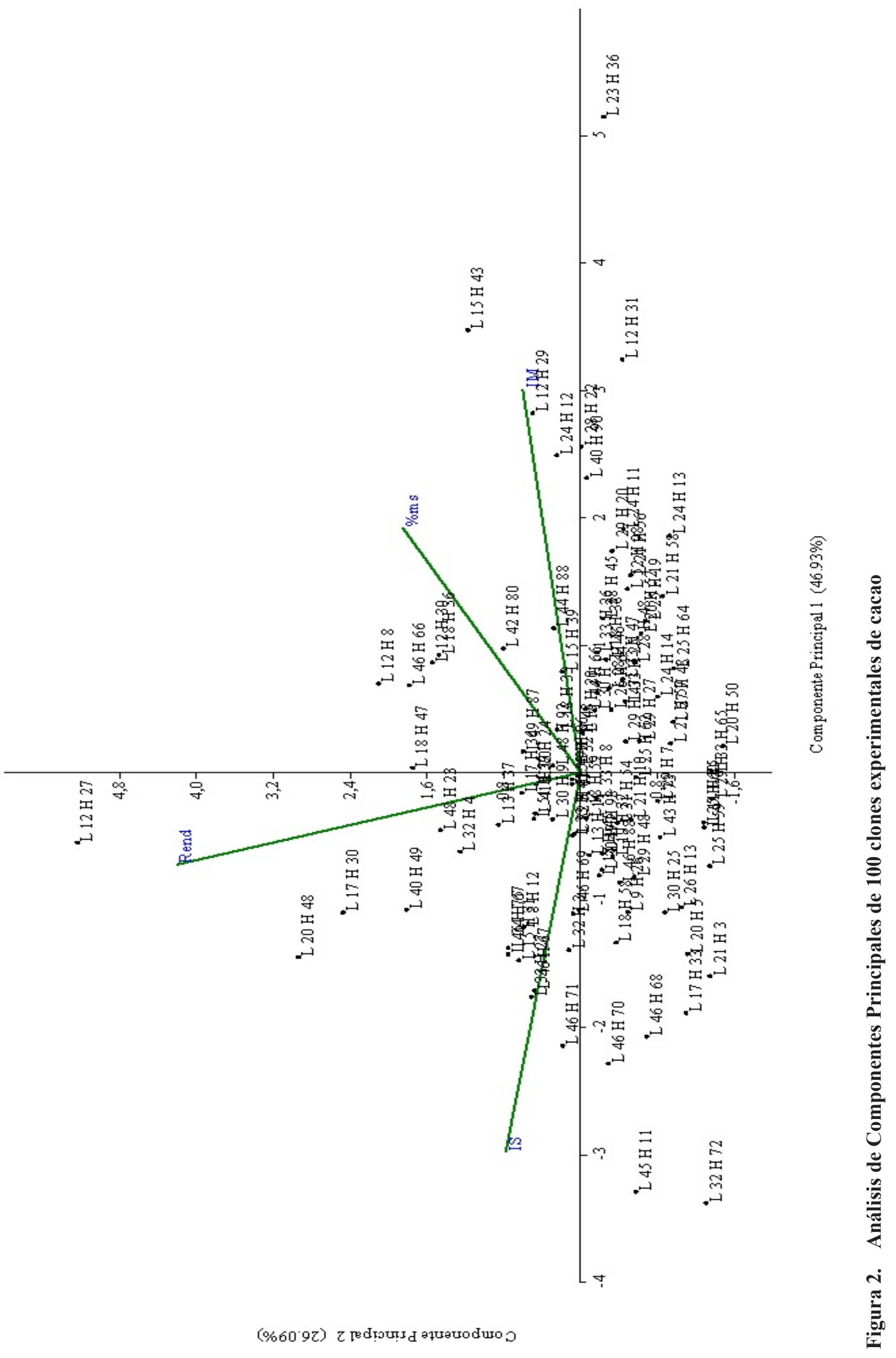


grupos, mostrando que los clones evaluados presentan diferencias en las características de producción y sanidad (Figura 2).

Al evaluar la variabilidad intra clon del porcentaje de mazorcas sanas producidas por cada clon (Figura 3), se observó que a medida que el porcentaje de mazorcas sanas se incrementa la variabilidad intra clon disminuye $\left(r^{2}=0.614\right.$, Sy.x = 5.701). Esto denota una mayor estabilidad del clon.
En los clones L12H29, L12H30, L15H43, L18H47, L24H12, L28H22 y L30H09 se observó un porcentaje de mazorcas sanas $>94 \%$ con una variabilidad intra clon $<6 \%$ (grupo de clones delimitados por un círculo). En el clon L20H50 se observó una proporción de mazorcas sanas del $25 \%$ y una variabilidad intra clon $>61 \%$.

En los clones L20H48, L12H27 y L17H30 (demarcado con hitos rojos) se observó porcentajes de mazorcas sanas de 89,87 y $68 \%$, respectivamente.

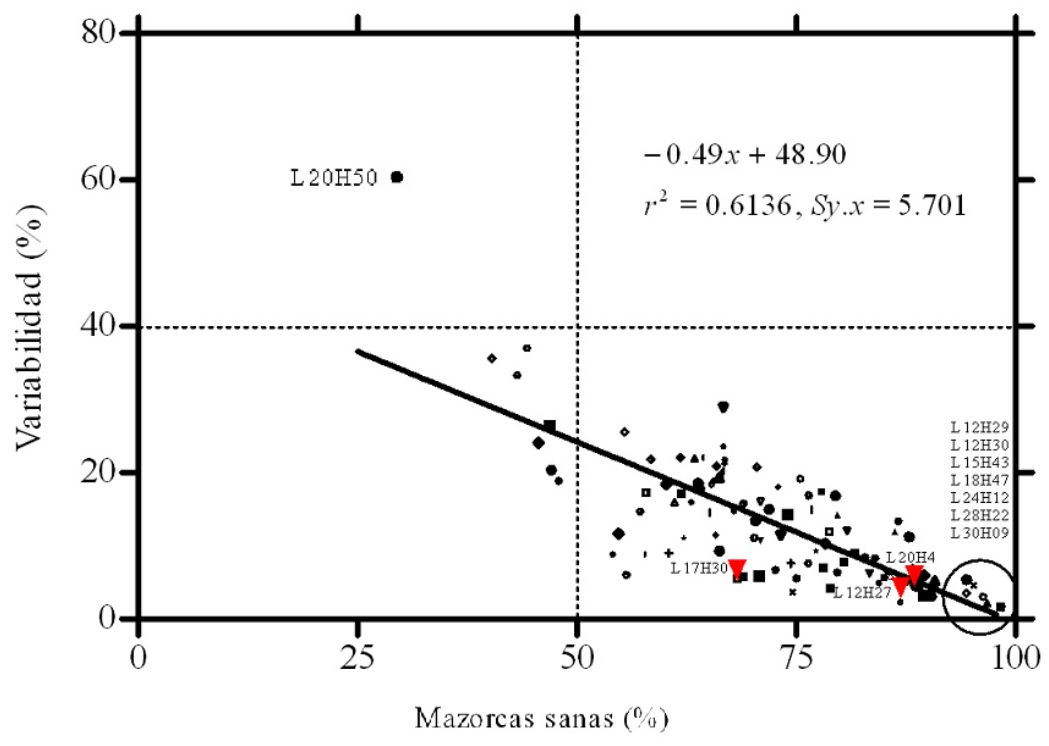

Figura 3. Porcentaje de mazorcas sanas de 100 clones experimentales de cacao tipo Nacional y su variabilidad intra clon

Sin embargo, fueron los tres clones que registraron los mayores rendimientos por hectárea, destacándose el clon L12H27 con $1820 \mathrm{~kg} \mathrm{ha}^{-1}$.

En la variable mazorcas sanas (\%) se destacaron los clones experimentales L12H27, L17H30 y L20H48, con 50, 21 y 19 mazorcas, respectivamente. Pérez (2009), registró una similar producción de mazorcas en los clones codificados como CATIE R-92 y CATIE R-70, con 19 y 17 mazorcas, respectivamente. Mientras que González y Vega (1992), evaluando clones experimentales obtuvieron un rendimiento promedio de 10 a 14 mazorcas por planta. Este comportamiento podría estar relacionado con las características genéticas de los genotipos evaluados (Phillips-Mora et al., 2009) y con los factores ecológicos determinantes en el desarrollo y producción de las plantas de cacao (Almeida y Valle, 2007; Omolaja et al., 2009).

Se observó que los clones experimentales L17H30 y L12H27 alcanzaron un rendimiento de 1154 y $1820 \mathrm{~kg}$ $\mathrm{ha}^{-1}$, respectivamente, con una variabilidad intra clon $<$ 20\% (Figura 4). El clon L20H48 alcanzó un rendimiento de $1092 \mathrm{~kg} \mathrm{ha}^{-1}$; sin embargo, la variación relativa superó ligeramente la media $(>41 \%)$. Los clones L17H30,
L12H27 y L20H48 fueron los más productivos.

De los 100 clones evaluados, en 97 se observaron rendimientos $<1000 \mathrm{~kg} \mathrm{ha}^{-1}$. No obstante, 61 de ellos con una variabilidad $<40 \%$ (cuadrante inferior izquierdo) y los 36 restantes con $>40 \%$ (cuadrante superior izquierdo).

La producción de estos clones experimentales se considera alta debido a que el rendimiento promedio de cacao a nivel de productor en el país es de $300 \mathrm{~kg}$ ha $^{-1}$ año $^{-1}$ (Quiroz y Amores, 2002). En un estudio Daymond et al. (2002). encontraron que el rendimiento potencial acumulado varió siete veces entre los clones EET-65 y ICS-8 con una producción equivalente al rendimiento de almendras secas de 200 y $1,389.0 \mathrm{~kg}$ ha $^{-1}$ año ${ }^{-1}$, respectivamente. Mientras que Pérez (2009) registró el mejor promedio de rendimiento de cacao con el clon CATIE R-92 con 1,441.0 kg ha ${ }^{-1}$ año $^{-1}$. Así también Sánchez-Mora et al. (2012), encontraron una mayor productividad en el clon experimental LR-16 con $1,126.8 \mathrm{~kg} \mathrm{ha}^{-1}$ año $^{-1}$. En este estudio, el desempeño productivo encontrado en clones de cacao tipo Nacional muestra una alta variabilidad genética que podría ser utilizada en programas de mejoramiento. 


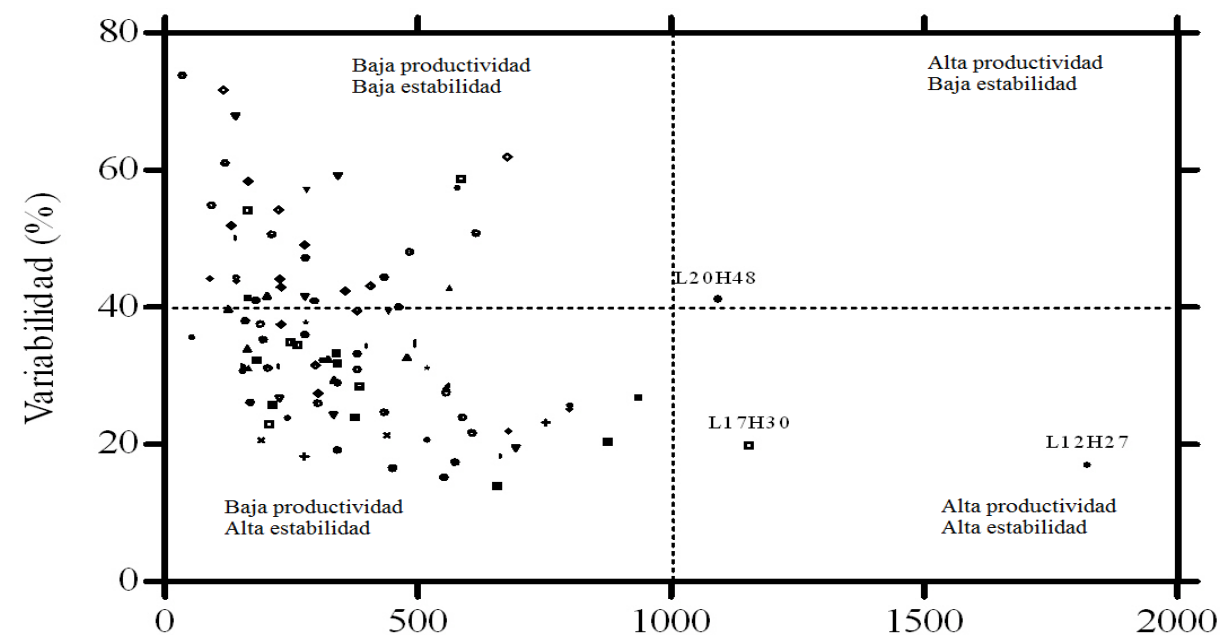

Rendimiento ( $\mathrm{kg} \mathrm{ha}^{-1}$ )

Figura 4. Rendimiento de 100 clones experimentales de cacao tipo Nacional y su variabilidad intra clon

\section{Conclusiones}

$\mathrm{L}$ a caracterización fenotípica de los clones experimentales de cacao tipo Nacional permitió establecer la existencia de una alta variabilidad genética entre los clones existentes en la finca experimental " $\mathrm{La}$ Represa".

Los clones de cacao conforman distintos grupos, lo cual indica que hay diferencias características de producción y sanidad.

Los clones seleccionados por alta productividad, adecuada estabilidad de la producción y cierta tolerancia a las enfermedades del cacao son: L12H27 y L17H30. El clon L20H48 requiere ser observado por un tiempo adicional para evaluar si mantiene el nivel alto de productividad y mejora su estabilidad ambiental.

Los clones experimentales tipo Nacional: L8H12, L9H26, L12H27, L12H29, L12H30, L13H11, L15H43, L17H30, L17H33, L18H45, L18H47, L18H58, L18H59, L19H32, L20H48, L20H5, L21H3, L24H12, L25H60, L28H22, L29H48, L30H09, L30H25, L30H7, L32H72, L33H27, L34H67, L41H70, L45H11, L46H67, L46H68, L46H70, L46H71, L46H75, L46H88 y $\mathrm{L} 48 \mathrm{H} 23$ representan una alta riqueza genética como germoplasma de cacao.

\section{Bibliografía}

Almeida, C.M.V.C., Dias, L.A.S., Silva, A.P. 2009. Caracterização agronômica de acessos de cacau. Pesquisa Agropecuária Brasileira 44(4): 368-373.

Almeida, A., Valle, R. 2007. Ecophysiology of the cacao tree. Brazilian Journal of Plant Physiology 19(4): 425-448
Arciniegas, A. 2005. Caracterización de árboles superiores de cacao (Theobroma cacao L.) seleccionados por el programa de mejoramiento genético del CATIE. Tesis Magister Scientiae. Centro Agronómico Tropical de Investigación y Enseñanza (CATIE). Turrialba. CR. 126 p.

Carvalho, C.G.P., Almeida, C.M.V.C., Cruz, C.D., Machado, P.F.R. 2001. Avaliação e seleção de híbridos de cacaueiro em Rondônia. Pesquisa Agropecuária Brasileira 36(8): 1043-1051.

Dantas-Neto, A., Corrêa, R.X., Monteiro, W.R., Luz, E.D.M.N., Gramacho, K.P., Lopes, U.V. 2005. Caracterização de uma população de cacaueiro para mapeamento de genes de resistência à vassoura-debruxa e podridão parda. Fitopatologia Brasileira 30:380-386.

Daymond, A.J., Hadley, P., Machado, R.C.R., Ng, E. 2002. Genetic variability in partitioning to the yield component of cacao (Theobroma cacao L.). HortScience 37(5): 799-801.

Enríquez, G. 2004. Cacao orgánico: guía para productores ecuatorianos. INIAP. Quito Ecuador. 359 p.

Evans, H.C. 2007. Cacao diseases - the trilogy revisited. Phytopathology 97: 1640-1643.

González, L.C., Vega, E.V. 1992. Evaluación de la reacción a moniliasis en clones e híbridos de cacao en Rio Frio. Costa Rica. Agronomía Costarricense 16(1): 13-22.

Grisales, S., Afanador, L. 2007. Análisis de variabilidad genética en Moniliophthora roreri con AP PCR y RAPD en Antioquia Colombia. Revista colombiana de biotecnología 9(2): 15-32. 
Hammer, Ø., Harper, D.A.T. Ryan, P.D. 2001. PAST: Paleontological Statistics software package for education and data analysis. Palaeontologia Electronica 4(1): 9pp. Site: palaeo-electronica. org/2001_1/past/issue1_01.htm

Leal, J.B., Santos, L.M., Santos, C.A.P., Pires, J.L., Ahnert, D., Corrêa, R.X. 2008. Diversidade genética entre acessos de cacau de fazendas e de banco de germoplasma na Bahia. Pesquisa Agropecuária Brasileira 43(7): 851-858.

Maddison, A.C., Macías, G., Moreira, C., Arias, R., Neira, R. 1995. Cocoa production in Ecuador in relation to dry-season escape from pod rot caused by Crinipellis perniciosa and Moniliophthora roreri. Plant Pathology 44: 982-998.

Meinhardt, L.W., Rincones, J., Bailey, B.A., Aime, M.C., Griffith, G.W., Zhang, D., Pereira, G.A.G. 2008. Moniliophthora perniciosa the causal agent of witches' broom disease of cacao: what's new from this old foe?. Molecular Plant Pathology 9(5): 577-588.

Omolaja, S., Aikpokpodion, S., Vwioko, D. 2009. Rainfall and temperature effects on flowering and pollen productions in cocoa. African Crop Science Journal 17(1): 41-48

Peña, G. 2003. Características fenotípicas de clones de cacao (Theobroma cacao L.). Tipo Nacional del banco de germoplasma de la Estación Experimental Tropical Pichilingue. Tesis Ing. Agr. Universidad Técnica de Manabí. Portoviejo. EC. 99 p.

Pereira, J.L., Almeida, L.C.C., Santos, S.M. 1996. Witches' broom disease of cocoa in Bahia: attempts at eradication and containment. Crop Protection 15(8): 743-752.

Pérez, J. 2009. Evaluación y caracterización de selecciones clónales de cacao (Theobroma cacao L.) del programa de mejoramiento del CATIE. Tesis Magister Scientiae en Agricultura Ecológica. Centro Agronómico Tropical de Investigación y Enseñanza (CATIE). Turrialba. CR. 149 p.
Phillips-Mora, W., Castillo, J., Krauss, U., Rodríguez, E., Wilkinson, M.J. 2005. Evaluation of cacao (Theobroma cacao) clones against seven Colombian isolates of Moniliophthora roreri from four pathogen genetic groups. Plant Pathology 54: 483-490.

Phillips-Mora, W., Aime, M.C., Wilkinson, M.J. 2007. Biodiversity and biogeography of the cacao (Theobroma cacao) pathogen Moniliophthora roreri in tropical America. Plant Pathology 56: 911-922.

Phillips-Mora, W., Castillo, J., Arciniegas, A., Mata, A., Sánchez, A., Leandro, M., Astorga, C., Motamayor, J., Guyton, B., Seguine, E., Schnell, R. 2009. Overcoming the main limiting factors of cacao production in Central America through the use of improved clones developed at CATIE. Proceedings of the 16th International Cocoa Research Conference, COPAL; 16-21th November 2009, Bali, Indonesia. p. 93-99.

Quiroz, J., Amores, F. 2002. Rehabilitación de plantaciones tradicionales de cacao en Ecuador. Manejo Integrado de Plagas 63: 73-80.

Sánchez, L., Gamboa, E., Rincón, J. 2003. Control químico y cultural de la moniliasis (Moniliophthora roreri Cif \& Par) del cacao (Theobroma cacao L.) en el estado Barinas. Revista de la Facultad de Agronomía LUZ 20: 188-194.

Sánchez-Mora, F.D., Vera, J.F., Ramos, R.A., Kamphorst, S.H. 2012. Avaliação do desempenho agronômico e sanitário de clones de cacau (Theobroma cacao L.) na zona de Quevedo. Equador. Em: Anais do II Congresso Brasileiro de Recursos Genéticos. Belém do Pará. BR. Trabalho 456. (Resumen extendido).

Sánchez-Mora, F.D., Garcés-Fiallos, F.R. 2012. Moniliophthora roreri (Cif y Par) Evans et al., en el cultivo de cacao. Scientia Agropecuaria 3: 249-258. 